\title{
Endometrial Necrosis Following B-Lynch Suture for Uterine Atony
}

\author{
Wenying Li*, Wenzhi Qin \\ Department of Obstetrics and Gynecology, Aviation General Hospital, Beijing, China \\ Email: *muziwen9999@163.com, muziwen9999@hotmail.com
}

How to cite this paper: Li, W.Y. and Qin, W.Z. (2019) Endometrial Necrosis Following B-Lynch Suture for Uterine Atony. Open Journal of Obstetrics and Gynecology, 9, 1032-1038.

https://doi.org/10.4236/ojog.2019.97100

Received: June 20, 2019

Accepted: July 22, 2019

Published: July 25, 2019

Copyright $\odot 2019$ by author(s) and Scientific Research Publishing Inc. This work is licensed under the Creative Commons Attribution International License (CC BY 4.0).

http://creativecommons.org/licenses/by/4.0/

(c) (i) Open Access

\begin{abstract}
B-Lynch compression suturing was performed on a 30-year old primipara during emergency Cesarean section (CS). After CS, she developed a low-grade fever, a subinvolution and tenderness of the uterus, and a pronounced increase in the inflammatory markers. Antibiotics were altered according to bacterial cultures and drug sensitivity testing of the cervix. By 10 days postpartum, a diagnostic curettage was performed and released a foul-smelling liquid matter due to the substantial amount of heterogeneous material with gaseous echoes showed via ultrasonography. The inflammatory markers gradually returned to normal by 9 days post curettage. At 12-day post curettage, a foul-smelling purulent tissue was extruded spontaneously via the vagina and proved to be necrotic tissue on pathologic examination. Eighteen months after childbirth, the patient had not experienced a menstrual period or subsequent pregnancy and a small uterus without any evidence of an endometrium showed by ultrasonography.
\end{abstract}

\section{Keywords}

Cesarean Section, Infertility, Postpartum Care, Postpartum Hemorrhage, Postpartum Infections

\section{Introduction}

Postpartum hemorrhage ( $\mathrm{PPH})$ is the primary cause of maternal mortality worldwide and in China [1] [2] [3] [4]. As an effective, fertility-preserving treatment for PPH caused by uterine atony, the B-Lynch suture has been used for 20 years [1] [2] [3] [4]. Future fertility and the ability to become pregnant seem likely [5] [6]. Unfortunately, some rare, severe complications, such as persistent vaginal discharge, uterine necrosis, formation of synechia, and pyometra, have been reported and necessitated hysterectomy as the use of this technique 
[6] [7] [8] [9]. This study stated and discussed another unusual complication as endometrial necrosis after B-Lynch suture for uterine inertia during cesarean section (CS).

\section{Case Report}

A 30-year-old primipara with well-controlled gestational diabetes was in labor and admitted to the hospital at 37 gestational weeks. She underwent an emergency CS for fetal distress 6 hours after admission; a fetus was delivered and all remnants of the placenta were easily separated from the inner lining of the uterus; however, the uterus became hypotonic and mild but constant bleeding proved to be unresponsive to uterine massage and immediate administration of oxytocin by 10 units intravenous drip and 20 units uterine injection followed by 100 microgram Carbetocin intravenous injection and two-dosage uterine injection of 250 microgram Carboprost Tromethamine with 15-minute interval. Therefore, we performed a B-Lynch procedure (compression suturing) and uterine tone and bleeding improved with a total uterine bleeding of no more than 700 milliliters (ml).

During the following 4 days postpartum, this primipara developed a low-grade fever $\left(\leq 38^{\circ} \mathrm{C}\right)$ and a pronounced increase in the following inflammatory markers: white blood cell count (WBC) of 19.4 to $21.9 \times 10^{9} / \mathrm{L}$, neutrophil percentage (NEUT\%) of $90.5 \%-91.8 \%$, and serum C-reaction protein (CRP) of $157 \mathrm{mg} / \mathrm{L}$ (as Table 1 showed). Interestingly, she had no noticeable complaints or anemia except for a subinvolution and tenderness of the uterus. In this hospital, Cefuroxime Sodium was intravenously administrated as routine after emergency CS.

Table 1. Change of serum white blood cell count (WBC, $\left.\times 10^{9} / \mathrm{L}\right)$, neutrophil percentage (NEUT\%), serum C-reaction protein (CRP, mg/L), procalcitonin (PCT, ng/ml) and hemoglobin (HGB, g/L) after cesarean section (CS).

\begin{tabular}{cccccc}
\hline Days after CS & WBC $\left(\times 10^{9} / \mathrm{L}\right)$ & NEUT\% & CRP $(\mathrm{mg} / \mathrm{L})$ & PCT $(\mathrm{ng} / \mathrm{ml})$ & HGB $(\mathrm{g} / \mathrm{L})$ \\
\hline 1 & 21.6 & 90.5 & NA & NA & 117 \\
3 & 21.9 & 91.3 & NA & NA & 113 \\
4 & 19.4 & 91.8 & NA & NA & 119 \\
5 & 19.5 & 87.4 & 157 & 0.23 & 117 \\
7 & 20.2 & 86.6 & 106 & 0.15 & 117 \\
8 & 20.7 & 89.0 & 105 & NA & 124 \\
16 & 13.8 & 82.2 & 93 & NA & 110 \\
17 & 11.0 & 81.6 & 95 & 0.29 & 108 \\
19 & 9.0 & 72.5 & 63 & 0.4 & 113 \\
22 & 10.8 & 75.6 & 32 & 0.44 & 109 \\
24 & 5.1 & 71.6 & 57 & 0.36 & 106 \\
28 & 9.5 & 72.3 & 5 & 0.45 & 126 \\
35 & 7.9 & 68.0 & $<1$ & NA & 119 \\
\hline
\end{tabular}


At day-5 postpartum, antibiotics were altered to Ceftriaxone Sodium due to abnormal serum inflammatory markers; and then Levofloxacin was added according to bacterial cultures and drug sensitivity testing of the cervix which was tested as Escherichia coli. Because there was no improvement in uterine involution or inflammatory markers by 10 days postpartum, a transabdominal ultrasonography was performed showing the uterine cavity to be filled with a substantial amount of heterogeneous material with gaseous echoes. Diagnostic curettage released a foul-smelling liquid matter without tissue being scraped out. Antibiotics were changed to Imipenem and Metronidazole intravenous drip based on the second round of bacterial cultures from uterine cavity which was tested as Enterococcus faecalis. During postpartum period, this young woman appeared good condition with no signs of toxemia; indeed, the WBC, NEUT\%, and CRP gradually returned to normal by 9 days post curettage (now 19 days post CS) and antibiotics were withdrawn, although enlarged uterus continued and there was persistence of the same type of abnormal findings on ultrasonography.

At 12-day post curettage (22-day post CS), a foul-smelling mass of $15 \times 12 \times 4$ $\mathrm{cm}^{3}$ of apparent purulent tissue was extruded spontaneously via the vagina which was proved to be necrotic tissue by pathologic examination. The second ultrasound-guided intrauterine exploration surgery was performed with large amount of foul smelling yellow pus out and the depth of uterine cavity was 14 centimeters. The third round bacterial culture from extruded tissue was tested as Pseudomonas putida and Escherichia coli. An additional 3 days of intravenous Levofloxacin and Metronidazole were administrated after the second postpartum surgery. Because she appeared to be well and was otherwise asymotomatic, she was discharged 35 days post-CS. At this time, her uterus was measured on ultrasonography at $7.9 \times 6.2 \times 3.0 \mathrm{~cm}^{3}$ without any clear evidence of an endometrium. During admission period, she never had temperature above $38^{\circ} \mathrm{C}$ despite severe elevation of inflammatory markers. Ultrasonography performed 18 months after childbirth showed a small uterus without any evidence of an endometrium. She worried the adverse influence to her baby by multiple antibiotics and chose delactation since in hospital. During those 18 months the patient had not experienced a menstrual period or subsequent pregnancy.

The research has been approved by Ethic Committee of Aviation General Hospital and informed consent has been given by the patient (Figures 1-3).

\section{Discussion}

The management of postpartum hemorrhage must be as fast, simple, and conservative as possible. In case of medical treatment failure, the use of surgical procedures as compression suture is recommended. Due to the ease of implementation, B-Lynch suture has been disseminated worldwide. However, some complications have occurred [1] [2] [3] [4]. Our case is similar to a previous report. 9 Both women underwent a B-Lynch suturing for uterine atony and late, 


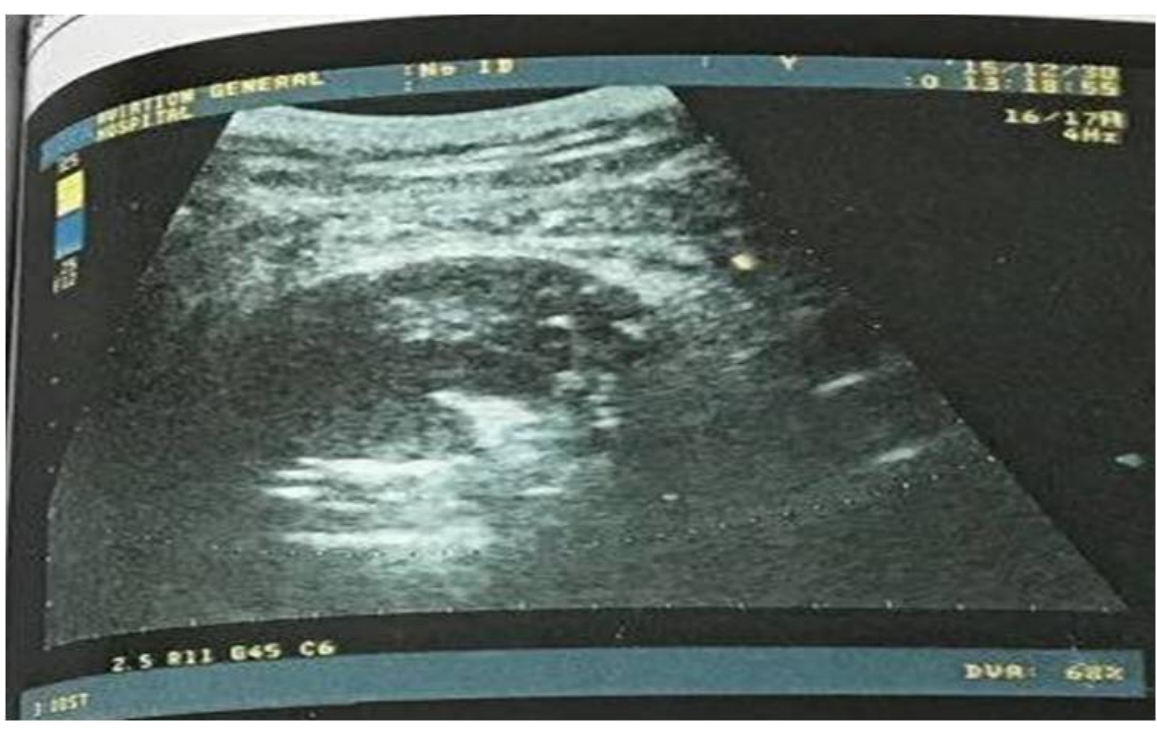

Figure 1. The image of transabdominal ultrasonography at 10 days postpartum.

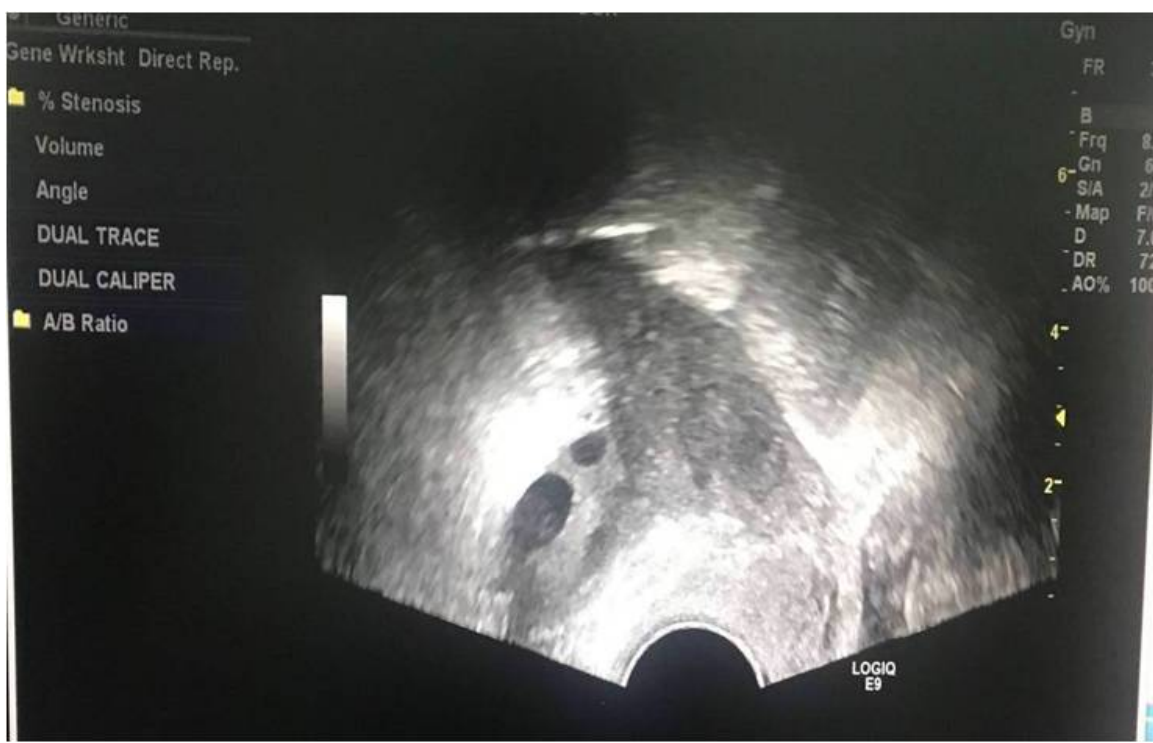

Figure 2. The image of transvaginal ultrasonography at 18 months postpartum.

persistent, bleeding of less than $1000 \mathrm{ml}$ during CS. Neither of these patients developed any noticeable uterine involution by 3 -week postpartum and uterine evacuation produced no findings, although there were abnormal intrauterine echoes on ultrasonography. Although our patient was treated without removing the uterus, the other patient underwent a hysterectomy at 2-month postpartum [9]. Some gynecologists may argue that the decision to place a B-Lynch suture may have been unwarranted for both women, because surgical intervention is usually suggested only for cases in which uterine massage, uterotonics, and balloon tamponade have failed [10]. Previous research has reported adoption of B-Lynch for blood loss of $300 \mathrm{ml}$, which may be conducted by younger, less experienced obstetricians [6]. The indication(s) for the B-Lynch suture should be re-visited, and surgical skills should be perfected via simulation training. 


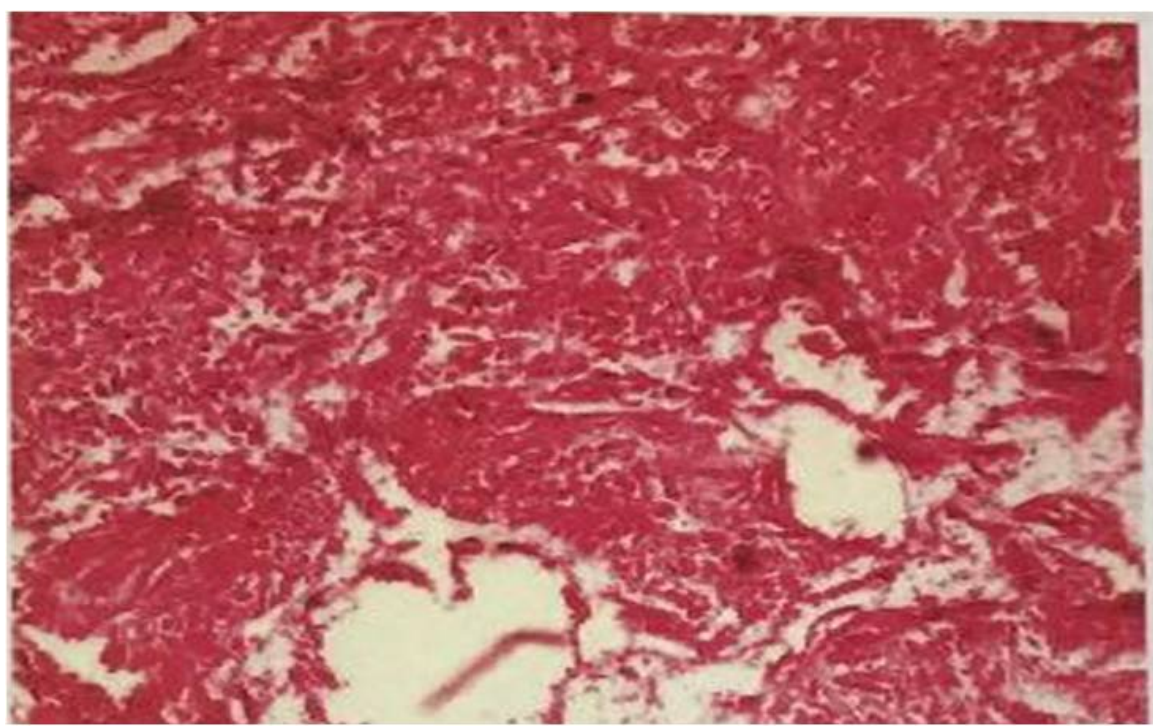

Figure 3. The image of tissue pathology at 22 days postpartum.

Our patient was very likely to be secondary amenorrhea though her uterus preserved. Severe postpartum hemorrhage at surgery may cause impairment of pituitary effects, which resulted in secondary amenorrhea. However, total blood loss was $700 \mathrm{ml}$ and hemoglobin was normal at day 1 after surgery in our case, which cannot support the effect of central system to the secondary amenorrhea. In situ due to severe endometrial infection might be the most reasonable explanation for amenorrhea. Ultrasound image showed a small uterus without any evidence of an endometrium which provided further evidence.

Unlike uterine necrosis, our patient suffered only endometrial necrosis after B-Lynch suturing. Despite her symptoms and signs being mild, there were only slight, inadequate uterine involution and pronounced increases in the numbers of inflammatory markers. Uterine necrosis is very rare because of the markedly increased collateral circulation of the pregnant uterus [8]. Overtight suture may compromise the uterine morphology, vasculature and lochia eduction. The hematoma within the uterus might increase intrauterine pressure and compress the blood supply to the endometrium and might explain the endometrial necrosis in this patient. Lack of involution of uterus and increases in inflammatory markers may alert the physician the possibility of uterine endometrial necrosis; moreover, this adverse outcome might be avoided by early use of ultrasonography, an exploratory laparotomy, and ablation of the uterine compression sutures and evacuation the hematoma. The use of antibiotics in our patient may not have been suitable. She was given 6 different families of antibiotics successively for a total of 25 days. There is consensus that prophylactic antibiotics should be used for no more than 24 hours postoperatively for an uneventful CS. We felt that the prolonged use of antibiotics in this patient was justified because of the abnormal increase in inflammatory marker and positive bacterial culture results. Nevertheless, the long-term use of antibiotics may very well have played an im- 
portant role in the uterine preservation, which was a great comfort to the parimipara and her husband, though the question of fertility persists. For this reason, the administration of broad-spectrum antibiotics may be an alternative to hysterectomy among parous women who suffer such a serious, complicated puerperal infection, despite the mild signs and symptoms.

In summary, our report shows that it is at least possible to preserve the uterus when the patient has endometrial necrosis after placement of a B-Lynch suture although the question of subsequent fertility remains. The lack of appropriate involution of the uterus accompanied by increases in inflammatory markers should be considered signs of either endometrial or uterine necrosis. Individualized considerations should be taken into account for hysterectomy which might be avoided by effective antibiotic administration and early use of ultrasonography and evacuation.

\section{Acknowledgements}

We appreciate the patient who allowed us to use her data and the Drs GuoLan Gao and LiQun $\mathrm{Yu}$ at the Aviation General Hospital for their comments on this manuscript.

\section{Disclosure}

We received no funding for this work. All the authors certify that they have participated sufficiently data collection, reference retrieval, and writing of the manuscript.

\section{Conflicts of Interest}

The authors have no competing interests to declare.

\section{References}

[1] Kayem, G., Kurinczuk, J.J., Alfirevic, Z., Spark, P., Brocklehurst, P. and Knight, M. (2011) Uterine Compression Sutures for the Management of Severe Postpartum Hemorrhage. Obstetrics and Gynecology, 117, 14-20. https://doi.org/10.1097/AOG.0b013e318202c596

[2] Kaya, B., Tuten, A., Daglar, K., Onkun, M., Sucu, S., Dogan, A., et al. (2015) B-Lynch Uterine Compression Sutures in the Conservative Surgical Management of Uterine Atony. Archives of Gynecology and Obstetrics, 291, 1005-1014. https://doi.org/10.1007/s00404-014-3511-2

[3] Enriquez, M., Maruri, G., Ezeta, G., Hidalgo, L., Perez-Lopez, F.R. and Chedraui, P. (2012) The B-Lynch Technique for the Management of Intraoperative Uterine Atony. Journal of Obstetrics and Gynaecology. The Journal of the Institute of $\mathrm{Ob}$ stetrics and Gynaecology, 32, 338-341. https://doi.org/10.3109/01443615.2012.673035

[4] Matsubara, S., Yano, H., Ohkuchi, A., Kuwata, T., Usui, R. and Suzuki, M. (2013) Uterine Compression Sutures for Postpartum Hemorrhage: An Overview. Acta $\mathrm{Ob}$ stetricia et Gynecologica Scandinavica, 92, 378-385.

https://doi.org/10.1111/aogs.12077 
[5] Tadakawa, M., Sugawara, J., Saito, M., Nishigori, H., Utsunomiya, H., Nagase, S., et al. (2015) Fertility and Pregnancy Outcomes Following B-Lynch Sutures for Post-Partum Hemorrhage. The Journal of Obstetrics and Gynaecology Research, 41, 559-564. https://doi.org/10.1111/jog.12590

[6] Fuglsang, J. (2014) Later Reproductive Health after B-Lynch Sutures: A Follow-Up Study after 10 Years' Clinical Use of the B-Lynch Suture. Fertility and Sterility, 101, 1194-1199. https://doi.org/10.1016/j.fertnstert.2014.01.015

[7] Liu, S., Mathur, M. and Tagore, S. (2014) Complications and Pregnancy Outcome Following Uterine Compression Suture for Postpartum Haemorrhage: A Single Centre Experience. Journal of Obstetrics and Gynaecology. The Journal of the Institute of Obstetrics and Gynaecology, 34, 383-386.

https://doi.org/10.3109/01443615.2014.895309

[8] Joshi, V.M. and Shrivastava, M. (2004) Partial Ischemic Necrosis of the Uterus Following a Uterine Brace Compression Suture. BJOG: An International Journal of Obstetrics and Gynaecology, 111, 279-280.

https://doi.org/10.1111/j.1471-0528.2004.00056.x

[9] Treloar, E.J., Anderson, R.S., Andrews, H.S. and Bailey, J.L. (2006) Uterine Necrosis Following B-Lynch Suture for Primary Postpartum Haemorrhage. BJOG: An International Journal of Obstetrics and Gynaecology, 113, 486-488. https://doi.org/10.1111/j.1471-0528.2006.00890.x

[10] Tuncalp, O., Souza, J.P. and Gulmezoglu, M. (2013) New WHO Recommendations on Prevention and Treatment of Postpartum Hemorrhage. International Journal of Gynaecology and Obstetrics. The Official Organ of the International Federation of Gynaecology and Obstetrics, 123, 254-256. https://doi.org/10.1016/j.ijgo.2013.06.024 